\title{
Distributed grating-assisted coupler for optical all-dielectric electron accelerator
}

\author{
Zhiyu Zhang, ${ }^{*}$ Sami G. Tantawi, and Ronald D. Ruth \\ Stanford Linear Accelerator Center, 2575 Sand Hill Road, Menlo Park, California 94025, USA
}

(Received 4 April 2005; published 18 July 2005)

\begin{abstract}
A Bragg waveguide consisting of multiple dielectric layers with alternating index of refraction becomes an excellent option to form electron accelerating structure powered by high power laser sources. It provides confinement of a synchronous speed-of-light mode with extremely low loss. However, laser field cannot be coupled into the structure collinearly with the electron beam. There are three requirements in designing input coupler for a Bragg electron accelerator: side coupling, selective mode excitation, and high coupling efficiency. We present a side-coupling scheme using a distributed grating-assisted coupler to inject the laser power into the waveguide. Side coupling is achieved by a grating with a period on the order of an optical wavelength. The phase matching condition results in resonance coupling thus providing selective mode excitation capability. The coupling efficiency is limited by profile matching between the outgoing beam and the incoming beam, which has normally a Gaussian profile. We demonstrate a nonuniform distributed grating structure generating an outgoing beam with a Gaussian profile, therefore, increasing the coupling efficiency.
\end{abstract}

PACS numbers: 41.75.Lx, 41.20.Jb, 41.60.Bq, 42.81.Qb

\section{INTRODUCTION}

The focus intensity of a high power laser has reached the levels of $10^{22} \mathrm{~W} / \mathrm{cm}^{2}$ [1], which corresponds to an electric field of $10^{14} \mathrm{~V} / \mathrm{m}$ in vacuum. The wall-plug efficiency of a high power laser system has been improved dramatically due to the rapid development of diode-pumped solid state laser. It therefore becomes increasingly appealing to utilize the intense electric fields available from high power laser pulses to drive charged particle accelerators. There are two types of vacuum laser accelerator being studied and they are both made of dielectric materials due to the relative high damage threshold and low loss at near-infrared laser wavelength. The LEAP [2] crossed laser beam accelerator represents the far-field scheme where particles are accelerated on the proximity of dielectric to satisfy the LawsonWoodward theorem $[3,4]$. The guided-mode scheme takes advantage of photonic crystals technology [5] confining propagating modes in vacuum through a band-gap guiding mechanism. Both 1D [6] and 2D [7,8] dielectric photonic crystal structures have been studied and a speed-of-light $\mathrm{TM}_{01}$ mode was found to be confined in the vacuum gap in these structures at near-infrared wavelength. The majority of laser power propagates in vacuum resulting in lower absorption loss and less detrimental nonlinear effects.

Coupling laser power into the accelerator structure by itself is a challenging subject. Waveguide and optical fiber input and output couplers have been studied extensively in the context of diode-pumped rare-earth-doped fiber lasers and amplifiers. Most of these fiber lasers are pumped by launching the pumping beam through the fiber end, i.e., end coupling. However, the end coupling scheme is not amenable to optical accelerator application because laser

*Electronic address: zhiyuz@SLAC.Stanford.EDU power cannot be coupled into the accelerator structure collinearly with charged particle beams. Moreover, it is difficult to increase the total pump power with the longitudinal end coupling scheme, which is limited ultimately by the damage threshold of the end face. Distributed side pumping schemes were developed to overcome these limitations. These techniques include angle-polished direct pumping $[9,10]$, embedded V-groove coupling [11], embedded mirror coupling [12], and prism coupling [13]. However, all of these techniques were designed for either double-cladding fiber or solid core photonic crystal fiber and cannot readily be used as a coupler for hollow core waveguides such as electron accelerators.

There are three first-order requirements to design an input coupler for an optical electron accelerator: (1) distributed side coupling; (2) selective mode excitation; (3) efficient power coupling. Side-coupling techniques for the fiber amplifier were not designed to excite a particular propagation mode in a waveguide except for prism coupling, in which case selective mode excitation can be achieved by choosing the incident angle and index refraction of the prism to satisfy the phase matching condition of a particular mode. However, a speed-of-light wave in the electron accelerator has a propagation constant $\beta=k_{0}=$ $2 \pi / \lambda_{0}$, where $\lambda_{0}$ is the vacuum wavelength. This mode is difficult to launch because total internal reflection occurs at the interface between the dielectric sidewall and the vacuum core if the phase matching condition is satisfied.

In this paper we propose a distributed grating-assisted coupler (DGAC) for optical electron accelerators to address these three requirements. Figure 1 shows the schematic diagram of a planar Bragg accelerator structure. The accelerator structure consists of a coupler region and an accelerating region. The DGAC in the coupler region couples the laser beam from the side and converts the laser 


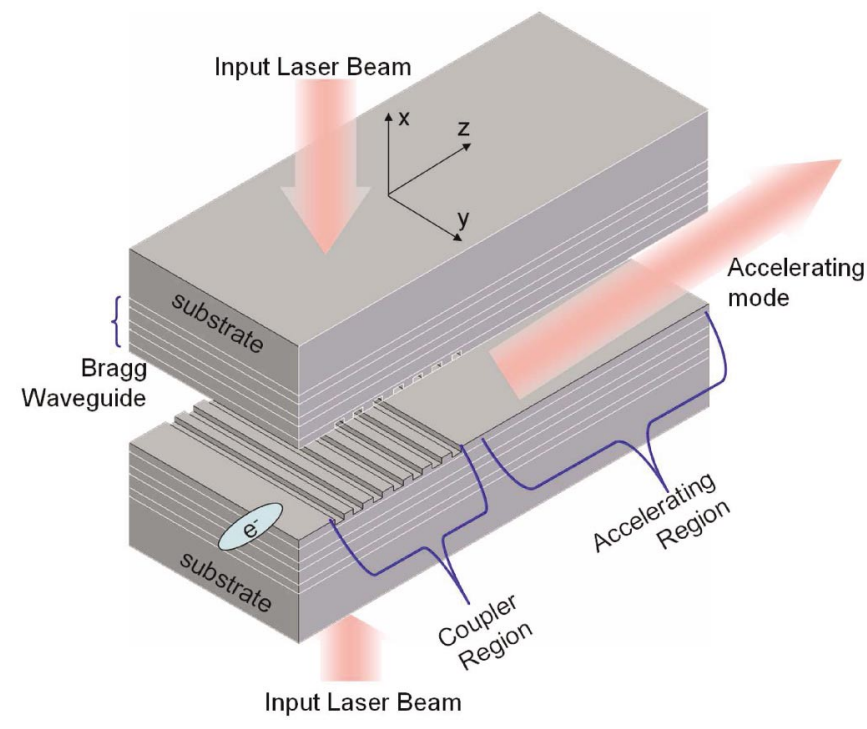

FIG. 1. (Color) Schematic diagram of a planar accelerator structure with distributed grating-assisted coupler (DGAC).

mode to the accelerating mode. This mode is guided by the planar Bragg waveguide in the accelerating region and copropagates with the electron beam. We only analyze the input coupler for a planar accelerator structure which confines the electromagnetic wave in one dimension $(x)$ in the following sections. However, the design principle discussed in this paper can be extended to the case of 2D ( $x$ and $y$ ) confinement.

\section{PLANAR ACCELERATOR STRUCTURE}

The theory of periodical layered waveguide structure, or Bragg fiber, was developed by Yeh [14] in the 1970s, and the fabrication of the Bragg fiber was first demonstrated by Fink et al. almost 30 years later [15]. Mizrahi and
Schächter extended the application of Bragg waveguide structures to optical electron accelerator [6].

Figure 2 shows the schematic diagram of the accelerating region of a planar Bragg accelerator structure. The electromagnetic field in the structure for a TM mode reads

$$
\begin{gathered}
E_{z}^{(i)}=\left(A_{i} e^{-j k_{t}^{(i)} x}+B_{i} e^{j k_{t}^{(i)} x}\right) e^{-j \beta z}, \\
H_{y}^{(i)}=-j \frac{n_{i}^{2} \omega \varepsilon_{0}}{k_{t}^{(i) 2}} \frac{\partial E_{z}^{(i)}}{\partial x} \\
=\frac{n_{i}^{2} \omega \varepsilon_{0}}{k_{t}^{(i)}}\left(-A_{i} e^{-j k_{t}^{(i)} x}+B_{i} e^{j k_{t}^{(i)} x}\right) e^{-j \beta z},
\end{gathered}
$$

where $k_{t}^{(i) 2}=n_{i}^{2} k_{0}^{2}-\beta^{2}, \beta$ is the propagation constant and $n_{i}$ the index refraction of the $i$ th layer. Coefficients $A_{i}$ and $B_{i}$ represent the electromagnetic field in a region where the dielectric constant is uniform. $A$ represents the amplitude of the outgoing wave and $B$ the amplitude of the incoming wave. Considering the boundary conditions that $E_{z}$ and $H_{y}$ are continuous at the $i$ th interface between dielectric layer $i$ and $i+1$, a $2 \times 2$ matrix can be found which relates $A_{i}, B_{i}$ to $A_{i+1}, B_{i+1}$ as

$$
\left[\begin{array}{c}
A \\
B
\end{array}\right]_{i+1}=M_{i+1, x_{i}}^{-1} M_{i, x_{i}}\left[\begin{array}{l}
A \\
B
\end{array}\right]_{i}
$$

where

$$
M_{i, x_{i}}=\left[\begin{array}{cc}
e^{-j k_{t}^{(i)} x_{i}} & e^{j k_{t}^{(i)} x_{i}} \\
-\frac{n_{i}^{2} \omega \varepsilon_{0}}{k_{t}^{(i)}} e^{-j k_{t}^{(i)} x_{i}} & \frac{n_{i}^{2} \omega \varepsilon_{0}}{k_{t}^{(i)}} e^{j k_{t}^{(i)} x_{i}}
\end{array}\right] .
$$

The synchronized mode in the vacuum region of the structure gives electromagnetic fields

$$
\left[\begin{array}{l}
E_{z} \\
H_{y}
\end{array}\right]_{1}=M_{1, x_{1}}\left[\begin{array}{l}
A \\
B
\end{array}\right]_{1}
$$

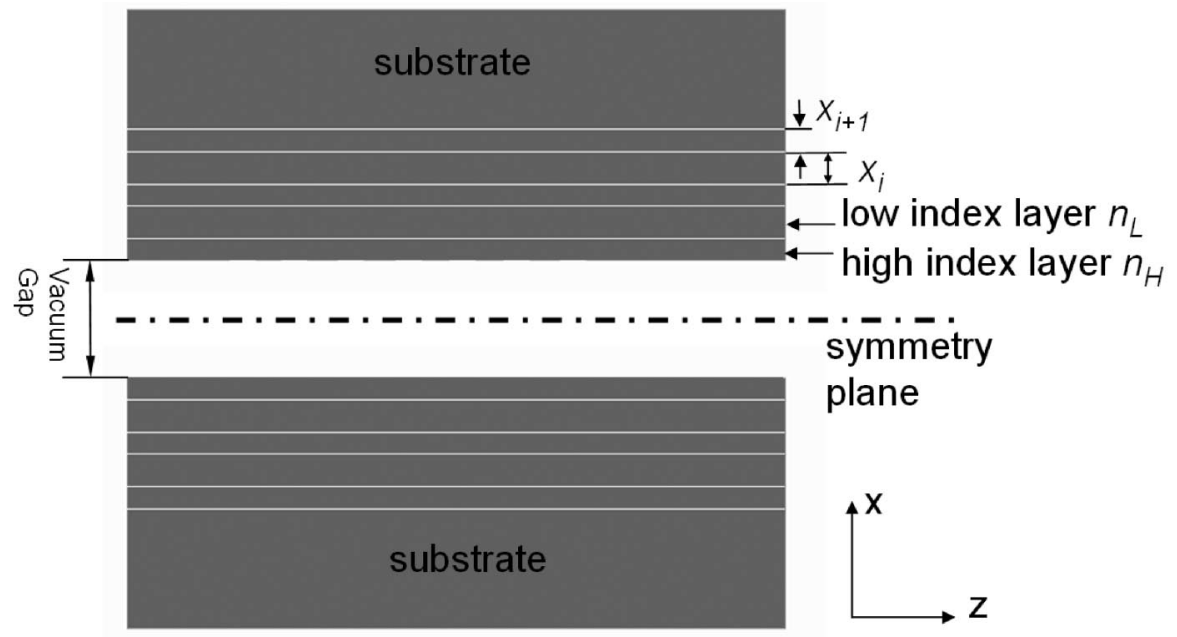

FIG. 2. Cross section of a planar accelerator structure ( $x-z$ plane of accelerating region shown in Fig. 1) consisting of layers with index of refraction $n_{H}$ or $n_{L}\left(n_{H}>n_{L}\right)$. The $i$ th layer has the thickness of $x_{i}$. 
where

$$
M_{1, x_{1}}=\left[\begin{array}{cc}
1 & 0 \\
0 & -j \omega \varepsilon_{0} x_{1}
\end{array}\right], \quad \text { and }\left[\begin{array}{l}
A \\
B
\end{array}\right]_{1}=\left[\begin{array}{l}
1 \\
1
\end{array}\right],
$$

which represents the uniform longitudinal electric field and linear increasing transverse field in the vacuum gap.

Wave confinement can be achieved by choosing the thickness of each layer to minimize the outgoing wave $A_{i}$ at the interface. Modes confined in a normal vacuum gap Bragg waveguide have a phase velocity exceeding the speed of light. However, the thickness of the first layer in an accelerator structure is not defined by the quarter wavelength thickness which other layers asymptotically approach; therefore, this layer can be viewed as a defect in the periodical structure. Coupling between the unperturbed propagating mode and the localized defect mode slows down the phase velocity to the speed of light in this structure. This can also be understood as impedance tuning effects [6]. Figure 3 shows the longitudinal electric field $\left(E_{z}\right)$ distribution of the confined acceleration mode in a waveguide consisting of alternating layers of $\mathrm{MgF}_{2}\left(n_{L}=\right.$ 1.36) and $\mathrm{TiO}_{2}\left(n_{H}=2.2\right)$. The wavelength is at $1064 \mathrm{~nm}$ and the vacuum gap is $1 \mu \mathrm{m}$. The $y$ axis overlaps with the symmetry plane shown in Fig. 2.

This multilayered dielectric structure shown in Fig. 3 is essentially a highly reflective dielectric mirror in a particular wavelength range. As shown in Fig. 4(a) the reflectivity of a normal incident beam is nearly $100 \%$ at the wavelength range between 1.2 and $1.5 \mu \mathrm{m}$; however, it is less than $5 \%$ at the design wavelength of $1064 \mathrm{~nm}$ labeled by a dashed line. The majority of a normal incidence laser beam is able to reach the innermost dielectric layer, where the DGAC converts the laser mode to the accelerating mode as illustrated in Fig. 1. Figure 4(b) presents the angular re-

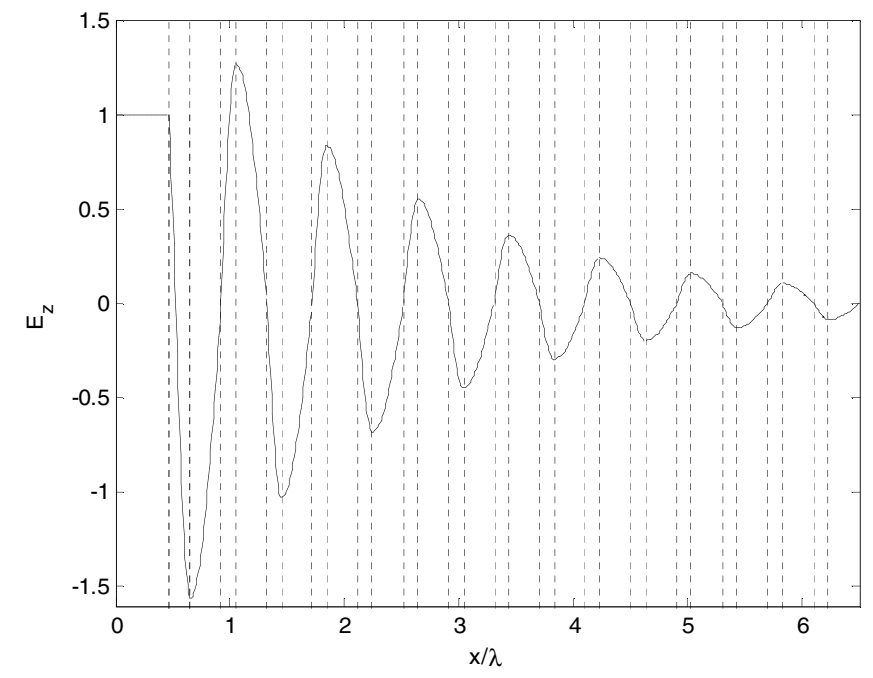

FIG. 3. Field distribution $\left(E_{z}\right)$ of a speed-of-light mode in a planar accelerator structure made of $\mathrm{TiO}_{2}$ and $\mathrm{MgF}_{2}$. The design wavelength is $1064 \mathrm{~nm}$ and the vacuum gap is $1 \mu \mathrm{m}$. Dashed lines are boundaries between dielectric layers.
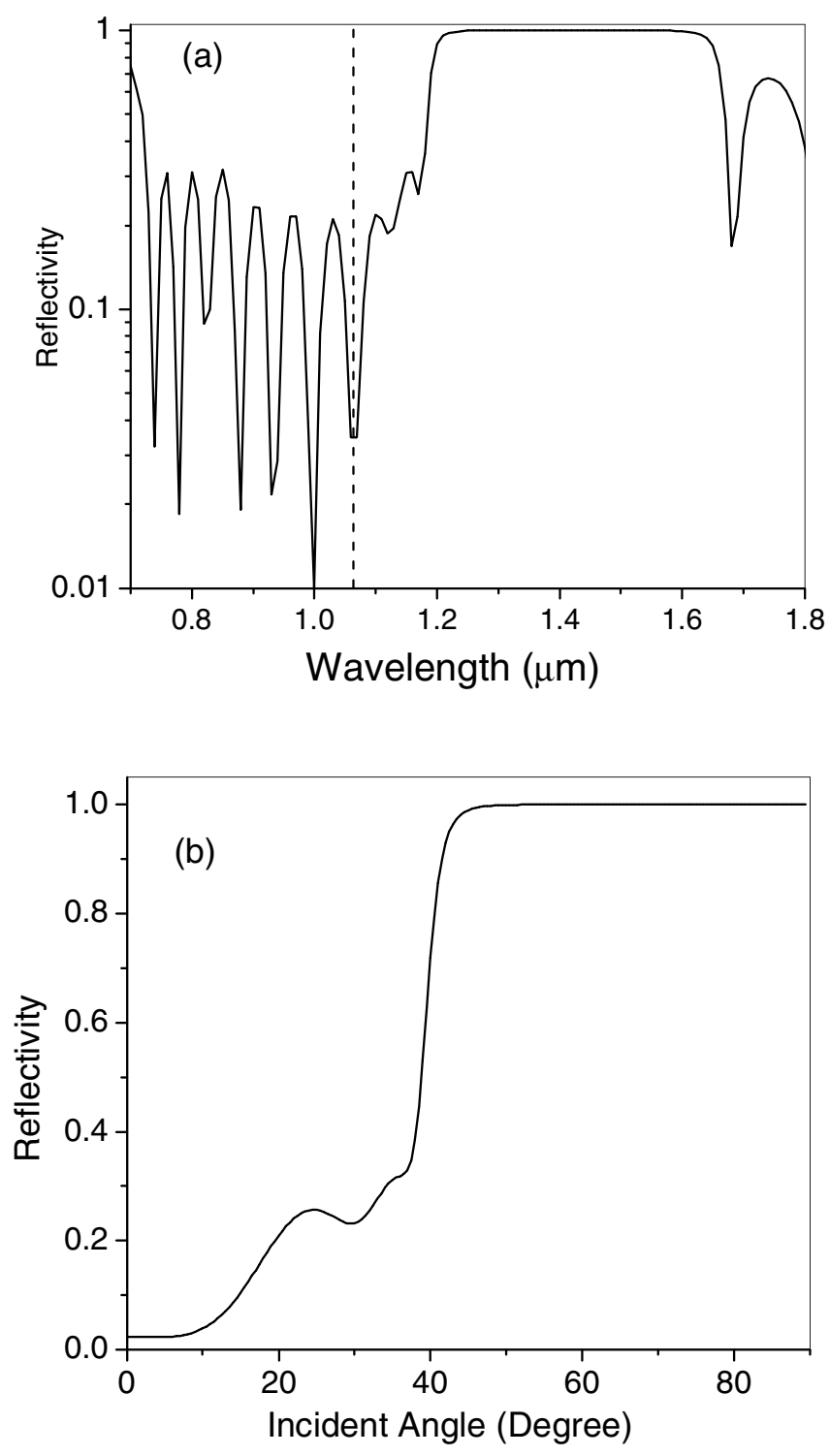

FIG. 4. Spectral characteristics of a planar accelerator structure shown in Fig. 3. Reflectivity as a function of (a) wavelength at normal incident angle (incident beam is along the $x$ axis in Fig. 2), design wavelength marked by a dashed line; (b) incident angle relative to the surface normal, i.e., $x$ axis in Fig. 2, at $1064 \mathrm{~nm}$.

sponse of this multilayered dielectric structure at design wavelength $1064 \mathrm{~nm}$. The incident angle is defined relative to the surface normal, or $x$ axis in Fig. 2. The reflectivity increases dramatically as the incident angle increasing above $10^{\circ}$. The angular response indicates that the inputcoupling angle, i.e., the incident angle of the laser beam, should be less than $10^{\circ}$ to achieve efficient side coupling for $\mathrm{TiO}_{2} / \mathrm{MgF}_{2}$ coating at $1064 \mathrm{~nm}$.

\section{BRAGG GRATING COUPLER}

Dielectric grating has found increasing applications as directional couplers because it can be integrated with 
waveguides using planar photolithographic techniques thus achieving stable and high efficiency performance. Extensive efforts have been devoted to the design, analysis, and fabrication of grating couplers [16-19].

Figure 5 shows a schematic diagram of a distributed grating-assisted coupler (DGAC) integrated on a planar slab waveguide. Optical power of a guided mode is confined within the slab waveguide by total internal reflection because the waveguide layer has a higher value of index of refraction than that of the substrate. The DGAC is fabricated on top of the slab waveguide and the function of it is to couple the optical power in or out of the waveguide plane as illustrated in Fig. 5 via the diffraction mechanism. The DGAC has a period on the order of an optical wavelength and its first-order diffraction beam is the desired out-of-plane output beam. Since the wave confinement of the slab waveguide is perturbed by the presence of the DGAC, the guided mode starts to leak out of the waveguide due to this perturbation; therefore, the output beam can be viewed as the leakage or radiation loss of the guided mode. The propagation constants of the output, or radiated beam, $\beta_{n}$ and guided beam in the slab waveguide $\beta$ are related to the grating period $\Lambda$ by the phase matching condition:

$$
\beta_{n} \sin \theta_{n}=\beta+n \frac{2 \pi}{\Lambda}
$$

and the radiated field varies as

$$
\exp \left[i\left(\beta_{n} \sin \theta_{n} z+i \alpha z-\omega t\right)\right]
$$

where $\beta_{n}$ is the propagation constant of the $n$th order of diffracted beam, $\Lambda$ is the grating period, $\beta$ is the propagation constant of a guided mode in the waveguide and it is close to the value obtained in the absence of the DGAC due to the weak perturbation induced by the DGAC, $\alpha$ is the radiation loss coefficient due to the leakage of the energy into the specific diffracted orders scattered by the DGAC, and $\theta_{n}$ is the $n$th order of diffraction angle. Detailed discussion of grating coupler on planar waveguide can be found in [20].

The phase matching condition can be further reduced in the case of an accelerating mode propagating in a Bragg dielectric waveguide, where $\beta=2 \pi / \lambda_{0}$ for an accelerating mode ( $\lambda_{0}$ is optical wavelength in air) and $\beta_{n}=$ $2 \pi / \lambda_{0}$ for a diffracted beam propagating in air. Equation (7) becomes

$$
\sin \theta_{n}=1+n \frac{2 \pi \lambda_{0}}{\Lambda} .
$$

Possible radiation beams have a real value of $\theta_{n}$. For example, given $\lambda_{0}=\Lambda$ and $n=-1, \theta_{-1}=0$, which means the output beam is perpendicular to the propagation direction of the input guided beam. This is the so-called resonance condition at which besides the out-of-plane beam second-order diffraction reflects straight back to the waveguide. Detuning the grating coupler away from the resonance condition is therefore desirable to achieve efficient coupling. Because of the grating term $n 2 \pi / \Lambda$ in Eq. (7), the grating coupler is able to selectively excite the acceleration mode satisfying the phase matching condition and avoid total internal reflection at the same time. The coupling angle can be tuned in a wide range by grating parameters and therefore the first two requirements of input coupler for the optical electron accelerator are naturally satisfied. In this paper, we designed and analyzed grating couplers for the planar accelerator structure using the CAMFR simulation tool based on eigenmode expansion and propagation technique and perfectly matched layers boundary conditions [21]. Only 1D gratings were considered, which were etched on the first dielectric layer of planar accelerator structure discussed in the previous section. It is easier for the mode expansion technique to calculate output coupling (from waveguide to air); however, the input-coupling efficiency can be readily calculated though the reciprocity principle. TM polarization (Efield normal to the grating groove and on the grating plane)

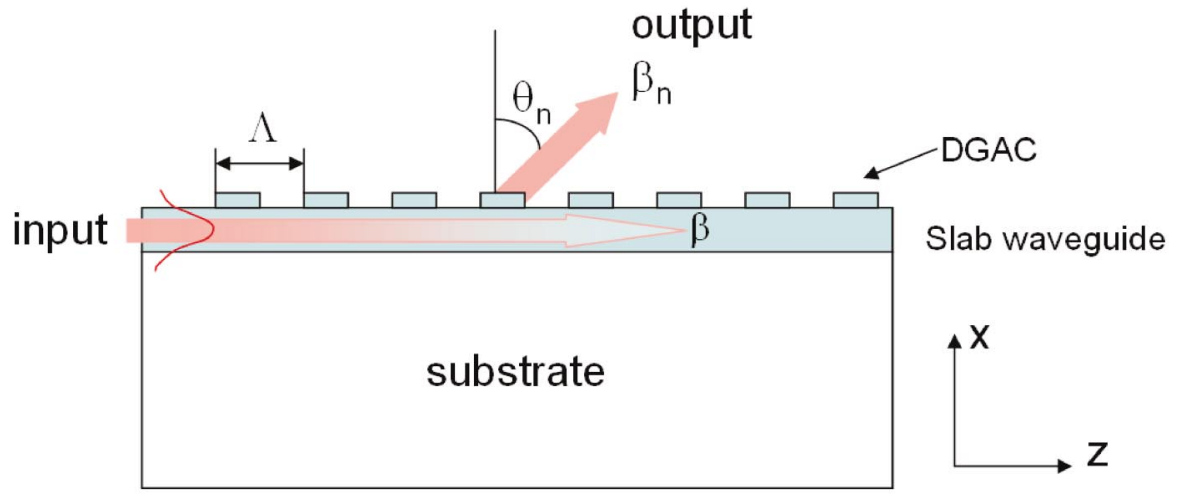

FIG. 5. (Color) Schematic diagram of a distributed grating-assisted coupler (DGAC) on a slab waveguide. This device becomes an out-of-plane coupler because the diffracted beam, or output beam, induced by the DGAC is not in the slab waveguide layer. $\beta$ is the propagation constant of a guided mode in the slab waveguide, $\beta_{n}$ is the propagation constant of the $n$th order of the diffracted beam, $\theta_{n}$ is the $n$th order of diffraction angle, and $\Lambda$ is the period of the DGAC. 


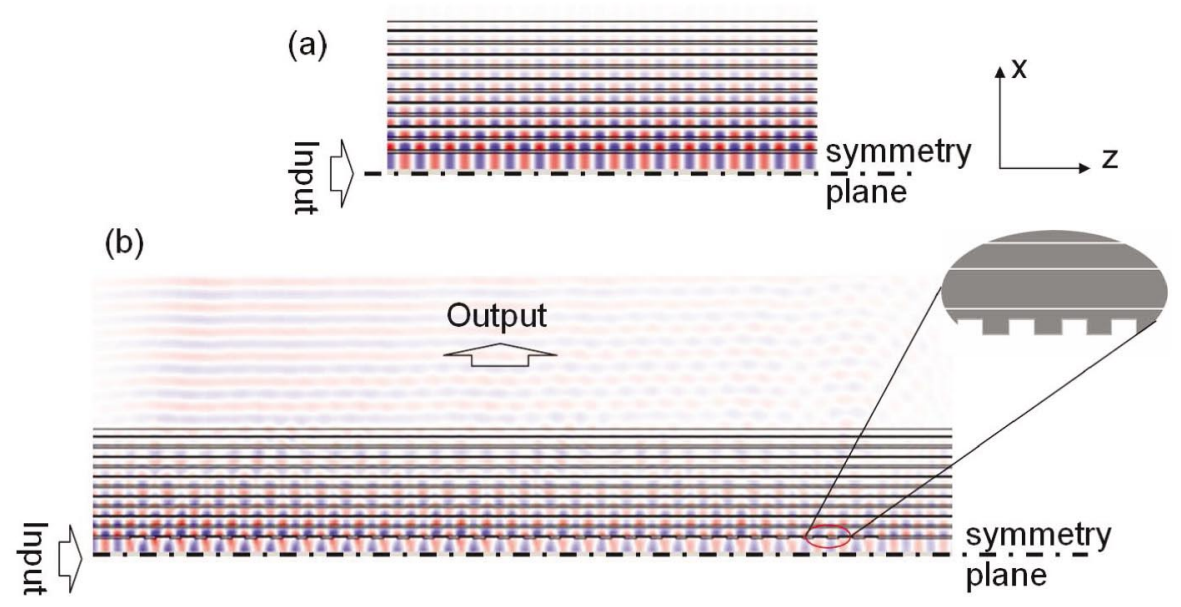

FIG. 6. (Color) Field plot $\left(E_{z}\right)$ of a dielectric electron accelerator (a) without the DGAC and (b) with the DGAC. Red and blue color represent positive and negative maximum values of the electric field. Shown here is half of the structure cut from the symmetry plane as shown in Fig. 2.

and design wavelength of $1064 \mathrm{~nm}$ were used throughout this paper.

Figure 6 shows the field plot $\left(E_{z}\right)$ of light in the Bragg waveguide accelerator. Shown here is the $x-z$ cross section of the structure illustrated in Fig. 1. The material $\left(\mathrm{TiO}_{2} / \mathrm{MgF}_{2}\right)$ and the thickness of each layer are the same as those presented in Fig. 3 designed to confine the accelerating mode. Figure 6(a) is the field plot of an accelerator structure in the absence of the DGAC. $E_{z}$ is well confined by 20 layers of alternating $\mathrm{TiO}_{2} / \mathrm{MgF}_{2}$ coatings. $E_{z}$ shown in Fig. 6(a) is uniform across the aperture, which is the characteristic feature of an accelerating mode. Figure 6(b) shows the field plot $\left(E_{z}\right)$ of accelerator structure with the DGAC, or the coupler region illustrated in Fig. 1. As shown in Fig. 6(b), the DGAC is etched on the first dielectric layer. The input beam, a confined synchronized mode, enters the coupler region from the left side. In the coupling region, because of the presence of the DGAC, the field is not confined by the waveguide anymore; rather, it couples to the radiated beam, which becomes a plane wave propagating along the $x$ axis. As of this particular example, the DGAC is made of rectangular grating grooves which are symmetrically etched on the first dielectric layer on both sides of the vacuum gap. The grating period is $1.08 \mu \mathrm{m}$ and the groove depth is $50 \mathrm{~nm}$ with $50 \%$ duty cycle. However, this resonance coupling $(\theta=0)$ demonstrated in Fig. 6(b) is not desirable and this effect can be understood with the help of Fig. 7.

Figure 7 represents the back reflection at the input port as a function of grating period with $50 \mathrm{~nm}$ groove depth. Strong back reflection occurs when the grating period is $1.08 \mu \mathrm{m}$, which corresponds to the resonance coupling as shown in Fig. 6(b). Slightly detuning from resonance condition, by either the grating period or groove depth, gives a higher coupling efficiency (up to $75 \%$ for a total of 30 periods).

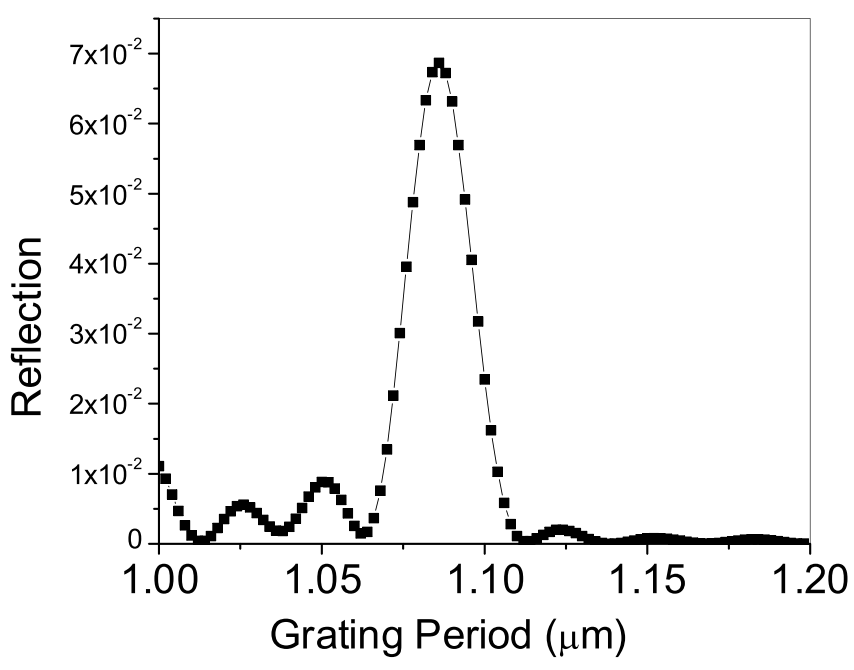

FIG. 7. Back reflection from the coupler region as a function of grating period.

\section{NONUNIFORM GRATING COUPLER}

In the previous section we analyzed uniform DGAC where the grating has a uniform period, groove depth, and duty cycle across the coupling region; therefore, the radiation loss coefficient $\alpha$ shown in Eq. (8) is a constant. The radiated beam has a characteristic exponential decay profile along the $z$ axis. Since laser output is normally a Gaussian profile, the intensity profile mismatch between the input and output beam results in a reduced inputcoupling efficiency. This effect can be analyzed using the reciprocity principle. The input-coupling efficiency is [20]

$$
\eta=P_{q} \frac{\left[\int g(z) h(z) d z\right]^{2}}{\int g^{2}(z) d z \int h^{2}(z) d z}
$$

where $g(z)$ and $h(z)$ represent the output and input intensity 
profiles, respectively, and $P_{q}$ is the output coupling efficiency. The input-coupling efficiency is therefore proportional to a normalized overlap function of input and output intensity profile. Considering the best case scenario of $P_{q}$ $100 \%$, and $g(z) \propto \exp (-\alpha z)$ where $\alpha$ is the radiation loss coefficient defined in Eq. (8), the maximum input-coupling efficiency is $80 \%$ for a Gaussian profile input $h(z) \propto$ $\exp \left[-\left(z-z_{0}\right)^{2} / \Delta^{2}\right], \quad$ where $z_{0}=0.5 / \alpha$ and $\Delta=$ $0.684 / \alpha$.

Several techniques have been developed to modulate the radiation loss coefficient along the propagation direction in an attempt to synthesize a desirable intensity profile for the planar waveguide input coupler. Using the first-order perturbation theory, Miyanaga et al. [22] showed that groovedepth linearly tapered sinusoidal gratings produce a nearGaussian profile. Touam [23] demonstrated using Green's function method and coupled-mode equation to synthesize symmetric a diffracted beam normal to a waveguide surface with a variable normalized grating duty cycle. Bates $e t$ al. [24] using a semiempirical method showed that an arbitrary beam profile can be synthesized by varying the groove depth of rectangular gratings. Since leakage rate is sensitive to the groove depth while the effect on the guided mode is minimal, side effects of nonuniform gratings such as variations in the coupling angle can be minimized. It is also relatively easier to fabricate groove-depth modulated nonuniform gratings as illustrated in [24]; therefore, we applied this technique to design a nonuniform DGAC for planar accelerator structure. Figure 8 illustrates a nonuniform DGAC where the groove depth of the grating is modulated. We will use this structure in the following section to demonstrate that a Gaussian output can be generated by this nonuniform DGAC.

We define field amplitude of a guided wave in the accelerator structure as $A(z)$ and it decays exponentially

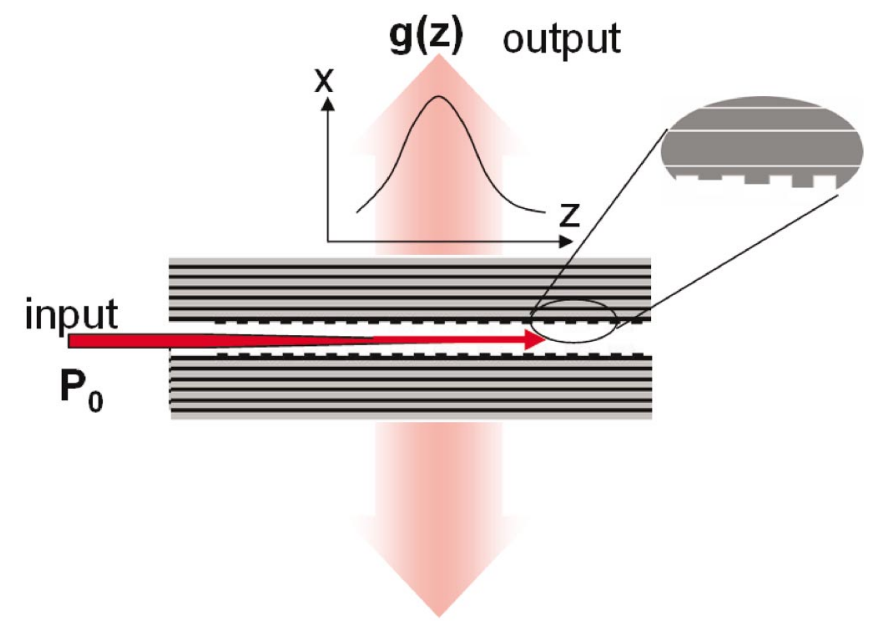

FIG. 8. (Color) A schematic diagram of a nonuniform DGAC where the grating groove depth is modulated to generate a Gaussian intensity profile $g(z)$ at the output, contrary to the exponential intensity profile generated by the uniform DGAC. due to radiation loss to the diffracted output beam, induced by the presence of the DGAC and it is characterized by the coefficient $\alpha$ :

$$
\frac{d A(z)}{d z}=-\alpha A(z)
$$

Radiated wave, or diffracted output beam is $B(z)$ and it is linearly related to $A(z)$ as

$$
B(z)=\beta A(z) .
$$

Both $\alpha$ and $\beta$ are constants in a uniform DGAC and both of them are a function of groove depth $h$. More specifically, $\alpha$ is quadratic and $\beta$ is linearly related to $h$ in a slab waveguide where fields are evanescent at the grating region; however, this is not the case for a grating coupler on planar accelerator structures. $\alpha$ and $\beta$ should be determined numerically by curve fitting. We started with a uniform DGAC with the period of $1.15 \mu \mathrm{m}$ and $50 \%$ duty cycle, which gives $3.5^{\circ}$ output coupling at $50 \mathrm{~nm}$ groove depth. Figure 9 shows $\alpha$ and $\beta$ vary as a function of
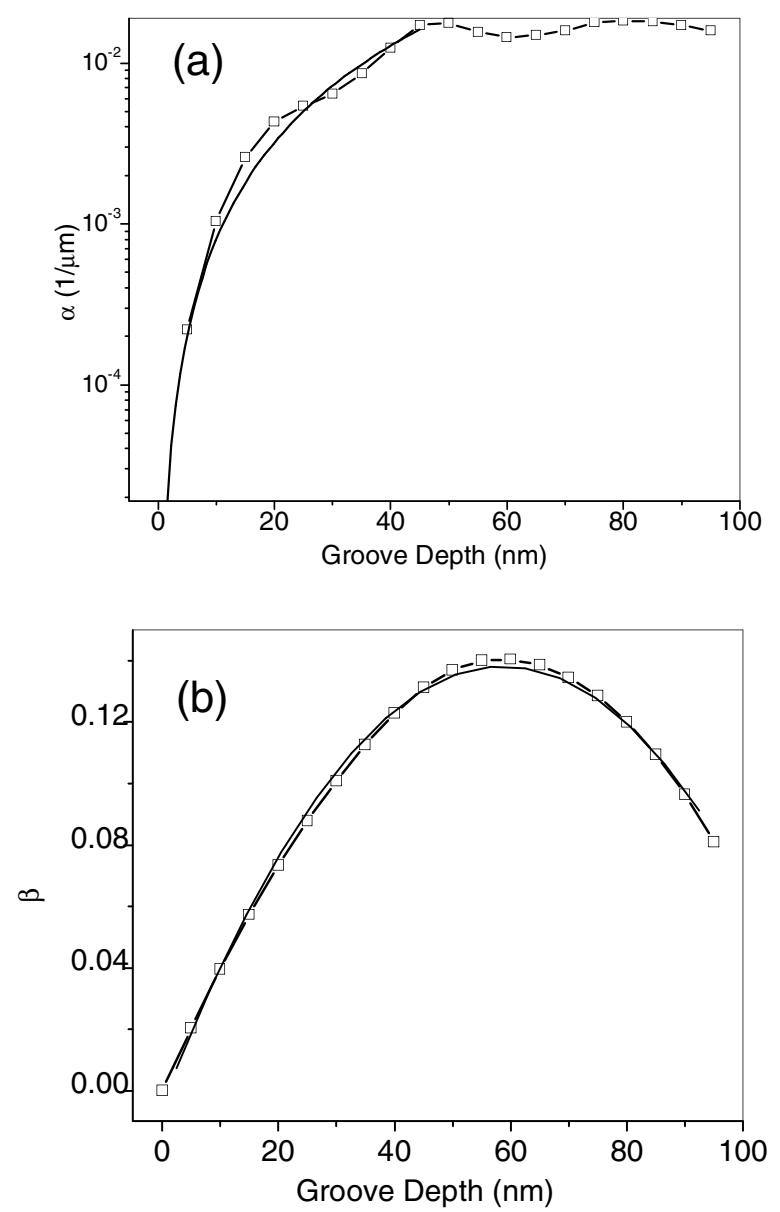

FIG. 9. (a) Radiation loss coefficient $\alpha$ and (b) ratio of the radiated and guided field $\beta$ as a function of grating groove depth $h$. The fitting results are also shown on this figure. $\alpha$ is fitted by $a h^{2}$ and $\beta$ is fitted by $b h+c h^{2}$. 


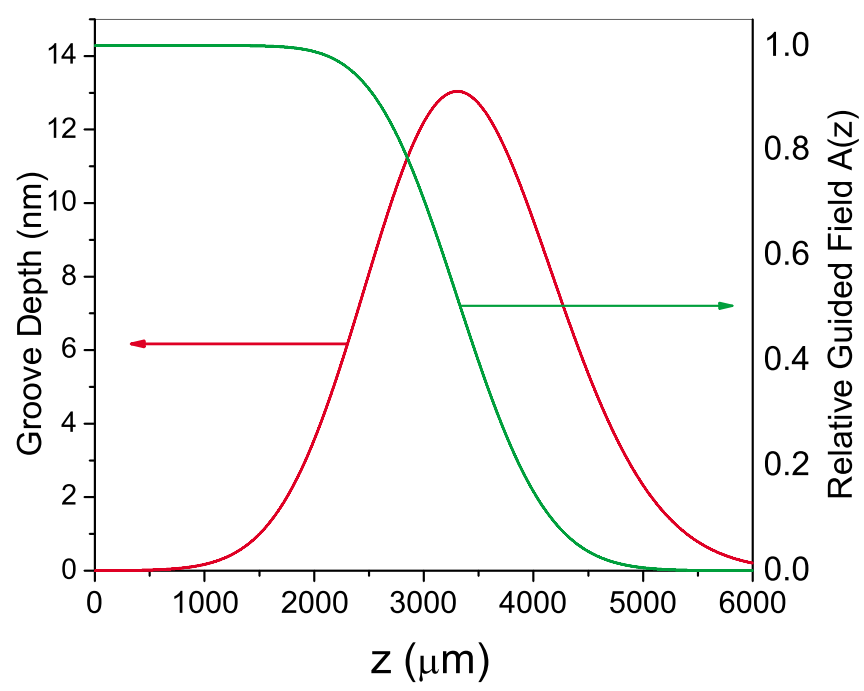

FIG. 10. (Color) Grating depth profile generating Gaussian output and the relative field strength of the guided wave.

groove depth. There are two features observed from this simulation. First, radiation loss coefficient $\alpha$ follows the parabolic rule only when groove depth is smaller than $50 \mathrm{~nm}$. Second, the ratio of radiated and guided power $\beta$ decreases for gratings with groove depth greater than $60 \mathrm{~nm}$. This is caused by the fact that as the groove depth increases, the perturbation induced by the presence of the DGAC becomes stronger and more optical power couples to the modes guided in the multilayered dielectric region by index guiding mechanism; therefore, the radiated power decreases as a result of it. For shallow grooves $(h<$ $50 \mathrm{~nm}), \alpha$ and $\beta$ are related to the groove depth $h$ by

$$
\begin{gathered}
\alpha=\mathrm{a} h^{2}(z), \\
\beta=b h(z)+\mathrm{ch}^{2}(z) .
\end{gathered}
$$

If applying the relations above to Eqs. (11) and (12), a groove-depth profile can be obtained for a given outcoupled wave profile $B(z)$. Figure 10 illustrates the groove-depth profile for a Gaussian output beam. The guided field $A(z)$ shown in the same figure is proportional to an error function. Although the radiation loss coefficient is rather small due to the shallow groove depth, the extended grating region (over $5 \mathrm{~mm}$ ) ensures that nearly $100 \%$ power is coupled out. The input-coupling efficiency, defined in Eq. (10), can approach $100 \%$ if using nonuniform grating coupler to match the input beam profile.

\section{FABRICATION}

The accelerator structure and the DGAC proposed in this paper can be fabricated using well-established technologies. Dielectric stack coatings can be fabricated with extremely high precision and low loss (see, for example, [25]) at our design wavelength. Coating materials are typically oxides and the damage threshold is comparable to fused silica, the substrate material; however, more work needs to be done on the effects of radiation damage on such materials. Because of its submicro feature size, the uniform DGAC structure should be patterned using either $e$-beam lithography or UV interference lithography. The grating pattern is then transferred on the first dielectric layer by dry etching techniques. Nonuniform DGAC can be fabricated using ion-beam etching [24]. Polysilicon or nitride can be deposited and pattered as a spacer layer to form vacuum gaps and it also serves as a bonding layer to bond two identical coated wafers. Finally, bonded wafers can be diced into individual accelerator structure for testing.

\section{CONCLUSION}

We have presented an all-dielectric Brag planar electron accelerator structure. A speed-of-light synchronous mode is confined in the structure with very low loss. The side coupling scheme is essential of distributed pumping the accelerator structure and recycling the residual power, and therefore is able to increase the overall coupling efficiency. Distributed grating-assisted coupler (DGAC) is proposed to couple laser light to the planar accelerator structure, and to selectively excite the synchronous accelerating mode. The coupling efficiency is optimized by matching the output and input intensity profile with modulated grating depth profile. The planar accelerator structures and gratings can be fabricated using well developed microprocessing technology.

\section{ACKNOWLEDGMENTS}

This research was funded by research grants from the U.S. Department of Energy under Contract No. DE-AC0276 SF00515.

[1] S.-W. Bahk, P. Rousseau, T. A. Planchon, V. Chvykov, G. Kalintchenko, A. Maksimchuk, G. A. Mourou, and V. Yanovsky, Opt. Lett. 29, 2837 (2004).

[2] R. Byer, T. Plettner, C. Barnes, E. Colby, B. Cowan, R. Siemann, and J. Spencer, in Proceedings of the Particle Accelerator Conference, New York, 1999 (IEEE, Piscataway, NJ, 1999), Vol. 1, p. 321.

[3] J. Lawson, IEEE Trans. Nucl. Sci. 26, 4217 (1979).

[4] P. Woodward, J. IEE 93, 1554 (1946).

[5] J. Joannopoulos, R. Meade, and J. Winn, Photonic Crystals: Molding the Flow of Light (Princeton University Press, Princeton, NJ, 1995).

[6] A. Mizrahi and L. Schächter, Phys. Rev. E 70, 016505 (2004).

[7] X. Lin, Phys. Rev. ST Accel. Beams 4, 051301 (2001).

[8] B. M. Cowan, Phys. Rev. ST Accel. Beams 6, 101301 (2003).

[9] J. Larsen and G. Vienne, Opt. Lett. 29, 436 (2004). 
[10] J. Xu, J. Lu, G. Kumar, J. Lu, and K. Ueda, Opt. Commun. 220, 389 (2003).

[11] D. Ripin and L. Goldberg, Electron. Lett. 31, 2204 (1995).

[12] J. P. Koplow, S. W. Moore, and D. A. V. Kliner, IEEE J. Quantum Electron. 39, 529 (2003).

[13] T. Weber, W. Luthy, and H. Weber, Appl. Phys. B 63, 131 (1996).

[14] P. Yeh and A. Yariv, J. Opt. Soc. Am. 68, 1196 (1978).

[15] Y. Fink, D. J. Ripin, S. Fan, C. Chen, J. D. Joannopoulos, and E. L. Thomas, J. Lightwave Technol. 17, 2039 (1999).

[16] T. Tamir and S. Peng, Appl. Phys. 14, 235 (1977).

[17] A. Hardy, D. Welch, and W. Streifer, IEEE J. Quantum Electron. 25, 2096 (1989).

[18] N. Eriksson, M. Hagberg, and A. Larsson, IEEE J. Quantum Electron. 32, 1038 (1996).
[19] M.H. Lim, T. Murphy, J. Ferrera, J. Damask, and H. Smith, J. Vac. Sci. Technol. B 17, 3208 (1999).

[20] H. Nishihara, M. Haruna, and T. Suhara, Optical Integrated Circuits, Optical and Electro-Optical Engineering (McGraw-Hill, New York, NY, 1989), Chap. 4.

[21] P. Bienstman and R. Baets, Opt. Quantum Electron. 33, 327 (2001).

[22] S. Miyanaga and T. Asakura, Appl. Opt. 20, 688 (1981).

[23] T. Touam and S. Najafi, Appl. Opt. 36, 2554 (1997).

[24] K. A. Bates, L. Li, R. Roncone, and J. Burke, Appl. Opt. 32, 2112 (1993).

[25] C. Walsh, A. Leistner, J. Seckold, B. Oreb, and D. Farrant, Appl. Opt. 38, 2870 (1999). 